\title{
Composition chimique et dégradabilité enzymatique et in vitro d'espèces ligneuses arbustives utilisables par les ruminants dans les parcours extensifs de la Nouvelle Calédonie. I. Typologie
}

\author{
C. Corniaux 1 N. Durand 1 J.M. Sarraihl ${ }^{2}$ H. Guerin ${ }^{3}$
}

\section{Mots-clés}

Plante d'abroutissement - Valeur nutritive - Classification - Composition chimique - Digestibilité - Ruminant Parcours - Nouvelle Calédonie.

\begin{abstract}
Résumé
Cette étude, réalisée sur 45 espèces arbustives récoltées en Nouvelle Calédonie, a permis de mettre en évidence six groupes de ligneux fourragers caractérisés par leurs teneurs en éléments bruts et leurs dégradabilités enzymatiques et in vitro. Les espèces présentant des teneurs en matières azotées dégradables et des dégradabilités de la matière organique élevées sont les plus intéressantes sur le plan nutritionnel. Il s'agit notamment de Gliricidia sepium, de Leucaena leucocephala, d'Acalypha grandis, de Samanea saman, d'Albizia lebbeck, d'Erythrina sp., d'Acacia farnesiana et de Sesbania grandiflora. En revanche, les données pour Desmanthus virgatus et, dans une moindre mesure, Acacia ampliceps sont décevantes.
\end{abstract}

\section{- INTRODUCTION}

En Nouvelle Calédonie, comme dans la plupart des pays de la zone Pacifique (Australie, Indonésie), la production de viande bovine a été sensiblement réduite depuis 1986 en raison de la diminution des ressources fourragères $(16,25)$. La raison majeure de cette diminution résulte de la régression importante de Leucaena leucocephala, ou faux mimosa, parasité par le psylle (Heteropsylla cubana) (7). Les solutions envisagées associent l'utilisation d'insectes prédateurs (8) et le recours à des arbustes fourragers résistants au psylle (7). Parmi ceux-ci figurent certaines espèces locales ou exotiques dont la nature et le mode d'exploitation sont actuellement à l'étude.

Ce projet êtablit une première évaluation des espèces locales identifiées soit comme étant utilisées couramment ou accidentellement par le bétail domestique ou sauvage, soit appartenant aux familles de légumineuses fixatrices d'azote, afin de compléter la gamme des espèces à tester sur les plans agronomiques et zootechniques.

Quarante-cinq espèces ligneuses répondant à ces critères ont été identifiées (12) et, afin d'avoir une première approche de leur valeur alimentaire, des échantillons de chacune d'entre elles ont été prélevés.

Ce travail présente les résultats de leur analyse ainsi qu'une typologie basée sur la teneur en composés organiques et minéraux et

1. CIRAD-Elevage, Yort Laguerre, BP 186, Nouméa, Nouvelle Calédonie 2. CIRAD-Forêt, Montravel, BP 10 001, Nouméa, Nouvelle Calédonie 3. CIRAD-EMVT, Campus international de Baillarguct, BP 5035, 34032 Montpellier, France les mesures de dégradabilité enzymatique ou de digestibilité in vitro. Dans un second article seront proposées des équations de prédiction des digestibilités in vitro et enzymatiques établies à partir de ces données.

\section{MATERIEL ET METHODES}

\section{Les ligneux fourragers}

Quarante-cinq échantillons d'organes d'espèces ligneuses arbustives ont été récoltés en juillet et août 1993 (12). En fonction du stade phénologique des plantes, ils étaient constitués de feuilles ou de fleurs, voire de fruits (tableau I). La collecte a été effectuée dans le sud de la Nouvelle Calédonie (Païta), sur la côte Ouest et la côte Est ainsi que sur les îles Loyauté. Les échantillons ont été séchés à l'étuve pendant $48 \mathrm{~h}$ à $70^{\circ} \mathrm{C}$.

Les 45 ligneux fourragers appartiennent à 14 familles différentes. Seulement quatre d'entre elles comportent plus de trois espèces : les Mimosacées (18 échantillons), les Fabacées (9), les Caesalpiniacées (3) et les Euphorbiacées (4). On ne s'intéressera donc aux familles quie dans ces quatre cas.

\section{Les mesures}

\section{Les laboratoires d'analyse}

Trois laboratoires ont analysés nos échantillons; le premier en Nouvelle Calédonie (Laboralvire d'Analyse des Aliments du Bétail, noté LAAB), le second en France (laboratoire d'alimentation CIRAD-EMVT, Maisons-Alfort), le troisième en Allemagne (Institut für Tierernährung, Universität Hohenheim). 
TABLEAU I

\section{Espèces arbustives récoltées en Nouvelle Calédonie}

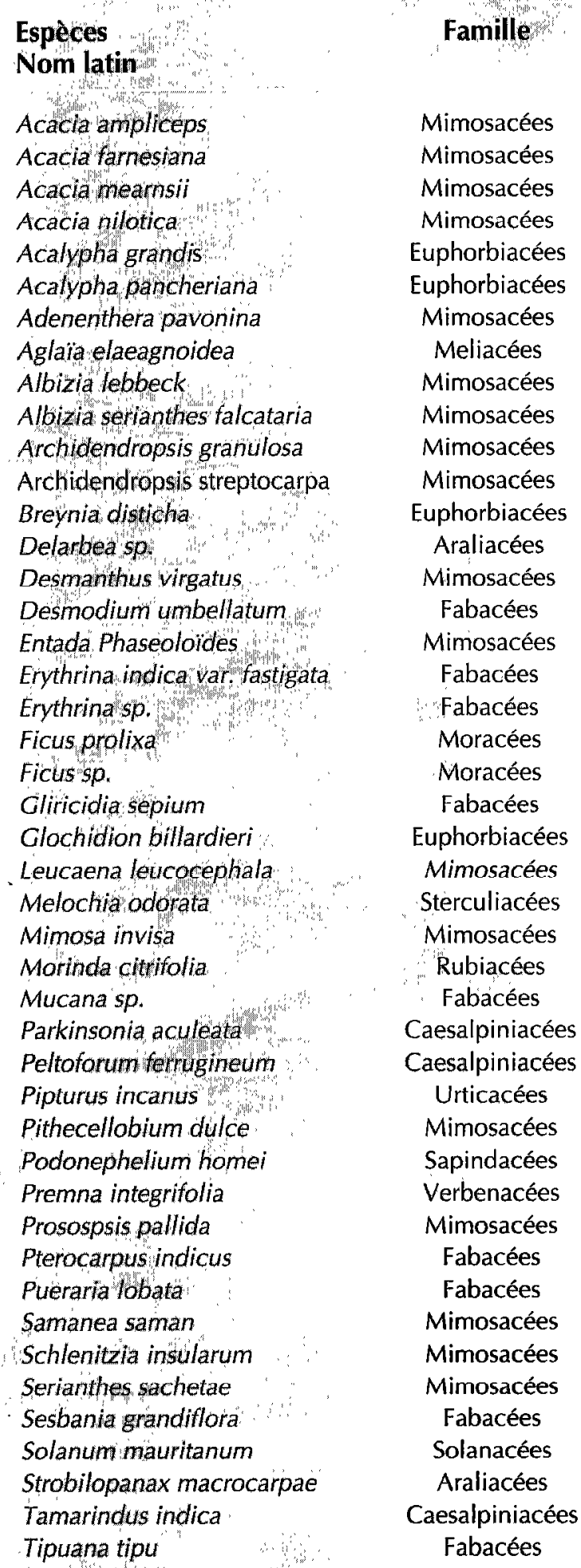

(1) feuilles vertes et tiges non chlorophyliennes

(2) cf. figure 5

Les analyses

Les principales méthodes d'analyses chimiques sont celles classiquement appliquées aux aliments du bétail et décrites par l'AFNOR (1) ou le BIPEA (6). Les références des autres méthodes sont indiquées ci-dessous.

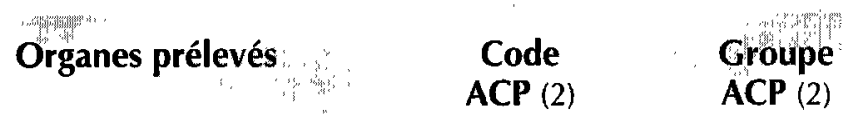

Feuilles, tiges (1)

Feuilles, tiges (1)

Feuilles, tiges (1)

Feuilles, tiges (1)

Feuilles, tiges (1)

Feuilles, tiges (1)

Feullies, tiges (1)

Feuilles, tiges (1)

Feuilles, tiges (1)

Feuilles, tiges (1)

Feuilles, tiges (1)

Feuilles, tiges ( 1 )

Feuilles, tiges, fleurs

Feuilles, tiges (1)

Feuilles; tiges, fleurs

Feuilles, tiges (1)

Feuilles, tiges (1)

Feuilles tiges (1)

Feuilies, tiges (1)

Feuilles, tiges (1)

Feuilles, tiges, fruits mûrs

Feuilles, tiges (1)

Feuilles, tiges (1)

Feuilles, tiges (1)

Feuilles, tiges (1)

Feuilles, tiges, fruits verts

Feuilles, tiges (1)

Feuilles tiges (1)

Feuilles tiges (1)

Feuilles ytiges (1)

Feuilles, tiges (1)

Feuilles, tiges (1)

Feuilles, tiges (1)

Feuilles, tiges (1)

Feuilles, tiges (1)

Feuilles, tiges (1)

Feuilles, tiges (1)

Feuilles, tiges (1)

Feuilles, tiges (1)

Feuilles, tiges (1)

Feuilles, tiges (1)

Feuilles, tiges (1)

Feuilles, tiges ( 1 )

Feuilles, tiges, fruits verts

Feuilles, tiges (1)
ACP (2)

ACP (2)

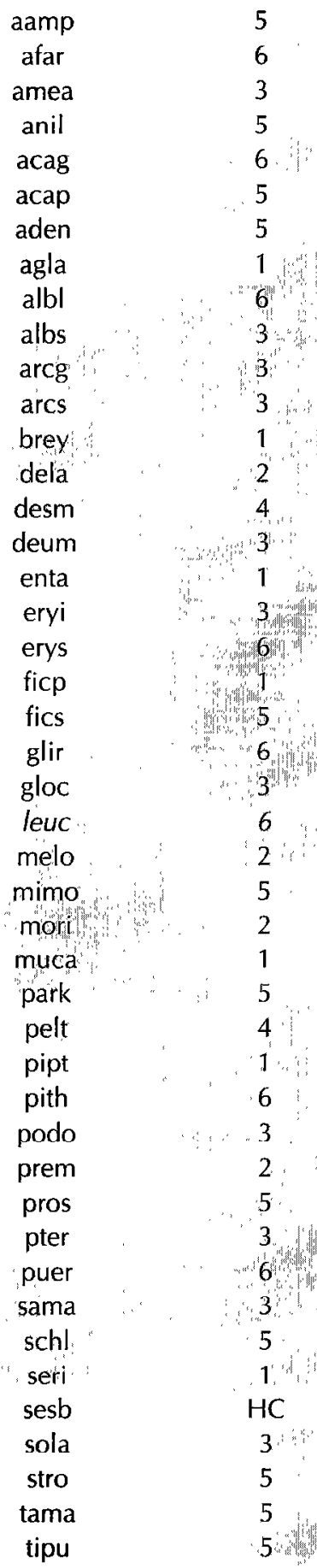

- Les critères chimiques déterminés sont :

- matière sèche, notée MS (p. 100 de la matière fraîche)

- matières minérales, notées MM (p. 100 MS)

- matières azotées totales, notées MAT (p. 100 MS)

- matières grasses, notées MG (p. 100 MS)

- cellulose brute, notée CB (p. $100 \mathrm{MS}$ ) 
- neutral detergent fiber, notée NDF (p. 100 MS) (26)

- acid detergent fiber, notée ADF (p. $100 \mathrm{MS}$ ) (26)

- acid detergent lignin, notée ADL (p. $100 \mathrm{MS}$ ) (26)

- calcium, noté $\mathrm{Ca}(\mathrm{g} / \mathrm{kg} \mathrm{MS})$

- phosphore, noté $\mathrm{P}$ (g/kg MS)

- MAadf : matières azotées liées à l'ADF (p. 100 MS) (20). Par différence, on obtient les matières azotées non liées à l'ADF (notées MAnadf).

- les teneurs en tanins précipitants et condensés (p. $100 \mathrm{MS}$ ) (13)

- Les critères enzymatiques mesurés sont:

- la dégradabilité enzymatique de la matière organique par la pepsine cellulase, notée SMO (p. 100 MS) (3)

- la dćgradabilité des matières azotées par la pronase après une heure d'incubation, notée DE1 (p. 100 MAT) (5). On obtient ainsi les teneurs en matières azotées dégradées par la pronase (notées MApro, p. $100 \mathrm{MS}$ ).

- Les critères de dégradabilité in vitro mesurés sont :

- la production de gaz mesurée lors du gastest de Hohenheim à partir de laquelle est estimée la digestibilité de la matière organique, notée dMOgt (p. $100 \mathrm{MS}$ ) (21) ainsi que les matières azotées dégradées in vitro (notées MAgt).

\section{L'analyse statistique}

\section{Statistiques élémentaires}

La moyenne, l'écart-type et le coefficient de variation ont été calculés pour les 15 variables suivantes : MS, MM, MAT, MAadf, $\mathrm{MG}, \mathrm{CB}, \mathrm{NDF}, \mathrm{ADF}, \mathrm{ADL}$, tanins précipitants, Ca, P, SMO, DE1 et dMOgt.

\section{Analyse de données}

- Choix de l'analyse de données

Nous disposons de données quantitatives qui peuvent être traitées par analyse en composantes principales (ACP). Elle nous permet de déterminer des groupes d'individus (espèces végétales) proches pour les variables prises en compte (teneur en éléments bruts et dégradabilités) et ainsi de présenter une typologie.

\section{-Choix des variables}

Les variables à prendre en compte dans l'ACP ont été sélectionnées à partir de la matrice de corrélations totales (choix de variables peu corrélées). Elles devaient également donner les informations les plus précises sur la valeur nutritionnelle des espèces arbustives à partir des critères chimiques, enzymatiques et de dégradabilité in vitro.

En ce qui concerne les critères chimiques, l'ADL, qui est indigestible, a été préféré pour caractériser les parois au NDF dont la digestibilité est variable suivant les proportions d'hémicellulose, cellulose et lignine qui le composent. La teneur en NDF des fourrages est en effet souvent considérée comme un meilleur prédicteur de l'ingestibilité (ou de l'encombrement) que de la digestibilité. Par ailleurs, sachant a priori que les MAT ne sont pas de bons estimateurs de la valeur nutritive des arbustes fourragers, on leur a adjoint un critère rendant mieux compte de leur fraction bloquée au niveau des parois végétales : MAadr. Les variables MM et MG n'ont, quant à elles, pas été retenues à cause de la possibilité de contamination exogène pour $\mathrm{MM}$ et, pour $\mathrm{MG}$, de son caractère non déterminant pour la valeur nutritive des ligneux fourragers. Enfin, la teneur en tanins précipitants, malgré son faible pouvoir prédicteur de la digestibilité, a été gardée dans l'analyse parce qu'elle est corrélée négativement avec les dégradabilités enzymatiques et in vitro et parce qu'elle joue un rôle sur l'appétabilité (par leur effet d'astringence qui ne peut être directement étudié en laboratoire).

Les dégradabilités enzymatiques de la matière organique (SMO) et des matières azotées (DE1) ont été retenues dans l'analyse. Le deuxième critère est notamment un bon estimateur de l'apport de matières azotées dégradables dans le rumen.

La dégradabilité in vitro de la matière organique (dMOgt), bien qu'elle soit très liée à la SMO, a été introduite dans l'analyse.

Ainsi, l'analyse de données est réalisée sur les sept variables suivantes:

- trois variables liées à la valeur azotée (MAT, MAadf et DE1)

- trois variables liées à la valeur énergétique (ADL, SMO et dMOgt)

- une variable relative aux facteurs antinutritionnels (tanins précipitants).

-Observations supplémentaires

Les familles de ligneux fourragers comportant plus de trois individus parmi les 45 récoltés et analysés ont été choisies comme observations supplémentaires. Il s'agit des trois familles de légumineuses (Mimosacées, Fabacées et Caesalpiniacées) et des Euphorbiacées.

RESULTATS

\section{Composition chimique et dégradabilité enzymatique et in vitro}

Les statistiques élémentaires (moyenne, coefficient de variation, minimum et maximum) figurent dans le tableau II.

TABLEAU $\|$

Statistiques élémentaires sur les 45 échantillons

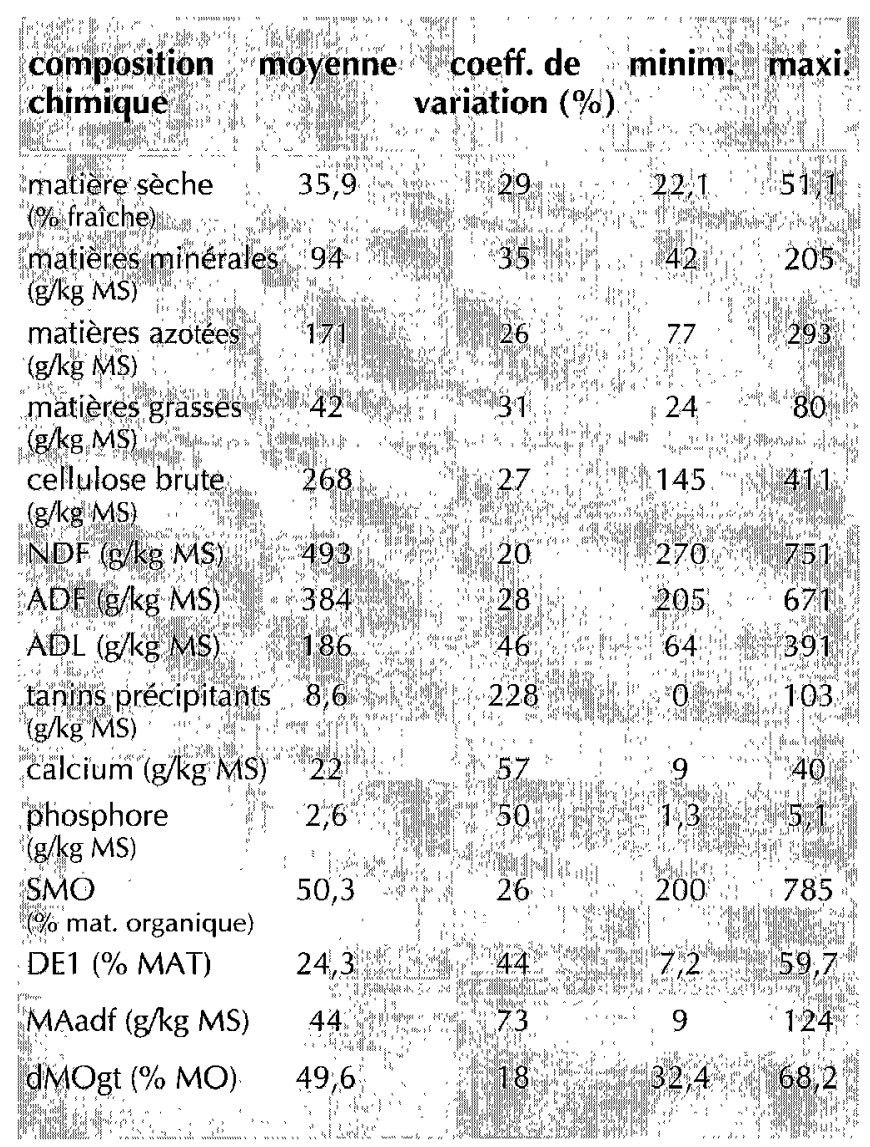


En moyenne, on peut noter notamment :

- une richesse relative en MAT (17 p. 100 MS) comparable à celle des légumineuses et supérieure à celle des graminées tropicales au stade "jeune repousse" (11 p. $100 \mathrm{MS})(9,19)$;

- une teneur en MAadf très variable (coefficient de variation de 77 p. 100) qui, comme pour les tanins $(\mathrm{CV}=228$ p. 100) est à mettre en parallèle avec la grande diversité botanique des ligneux (tableau I). Néanmoins, les espèces riches en MAT (> 20 p. 100 MS) ont généralement des teneurs faibles en MAadf, ce qui les rend d'autant plus intéressantes sur le plan nutritionnel. Parallèlement, les espèces riches en MAT le sont généralement en MAnadf (figure 1);

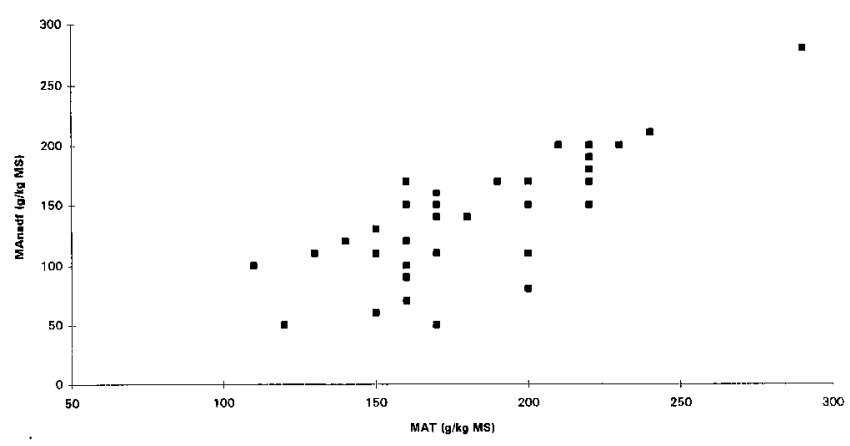

Figure 1: MAnadf fonction de MAT $(r=0,79)$.

- une dégradabilité de la matière organique de l'ordre de 50 p. 100 comparable à celle des pailles (15). Comme pour les fourrages herbacés, elle est négativement corrélée à la teneur en NDF (figure 2) ;

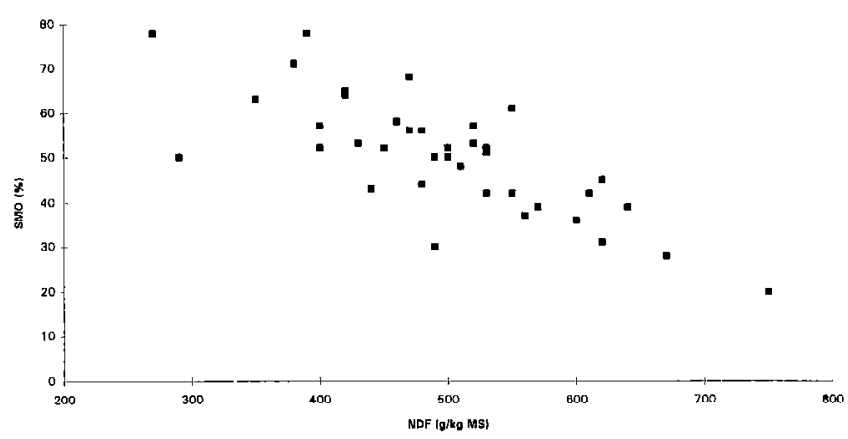

Figure 2 : SMO fonction de NDF $(r=-0,72)$.

- une dégradabilité enzymatique des matières azotées de l'ordre de 25 p. 100 ;

- une teneur en MAadf fonction de la teneur en ADL (figure 3) :

- globalement, une grande variabilité de la composition chimique et de la dégradabilité enzymatique. En particulier, on peut noter des coefficients de variation de 228 p. 100,73 p. 100,57 p. 100 et 46 p. 100 respectivement pour les teneurs en tanins précipitants, MAadf, calcium et ADL. Ces résultats sont d'ailleurs conformes aux travaux de Guerin (14) et Ickowicz (18).

\section{Typologie}

\section{Le plan principal}

Les pourcentages de variation expliqués par les axes principaux de l'ACP sont les suivants :

- axe $1: 43,3$ p. 100

- axe $2: 20,0$ p. 100

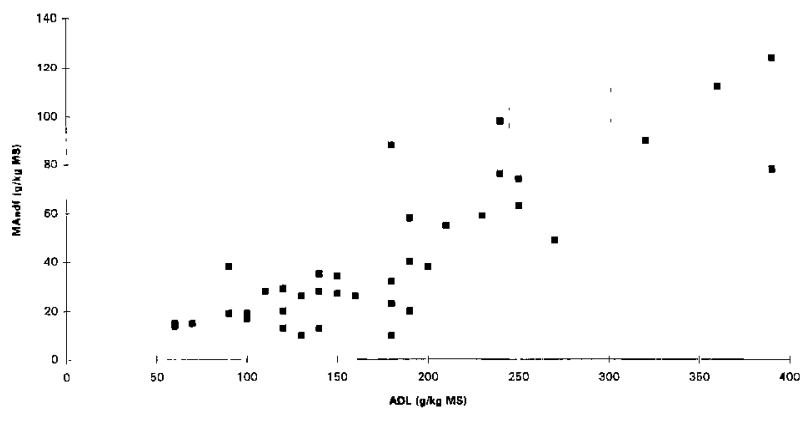

Figure 3 : MAadf fonction de $A D L(r=0,83)$.

- axe $3: 17,8$ p. 100

- axe $4: 9,3$ p. 100

- axe $5: 4,7$ p. 100

La part d'information expliquée par les axes 1 et 2, qui forment le plan principal, est donc de 63,3 p. 100.

Compte tenu de cette représentativité et' afin de ne pas compliquer la typologie, la projection des variables (teneurs en MAT, ADL, MAadf et tanins précipitants, SMO, dMOgt et DE1) et des observations (45 espèces ligneuses) ne sera étudiée que sur le plan principal.

\section{Les variables}

L'information représentée sur le plan principal est de :

- 90 p. 100 pour MAadf "très bien représentée" (24)

- 85 p. 100 pour ADL "très bien représentée"

- 80 p. 100 pour dMOgt "très bien représentée"

- 75 p. 100 pour SMO "bien représentée"

- 61 p. 100 pour DE1 "bien représentée"

- 37 p. 100 pour tanins précipitants "mal représentée"

- 14 p. 100 pour MAT "mal représentée".

La discrimination des observations projetées sur le plan principal se fera donc par les teneurs en ADL et en MAadf ainsi que par la digestibilité in vitro de la matière organique. Les valeurs DE1 et SMO interviennent dans une moindre mesure. Les teneurs en tanins précipitants et en MAT n'ont par contre qu'une très faible incidence (figure 4).

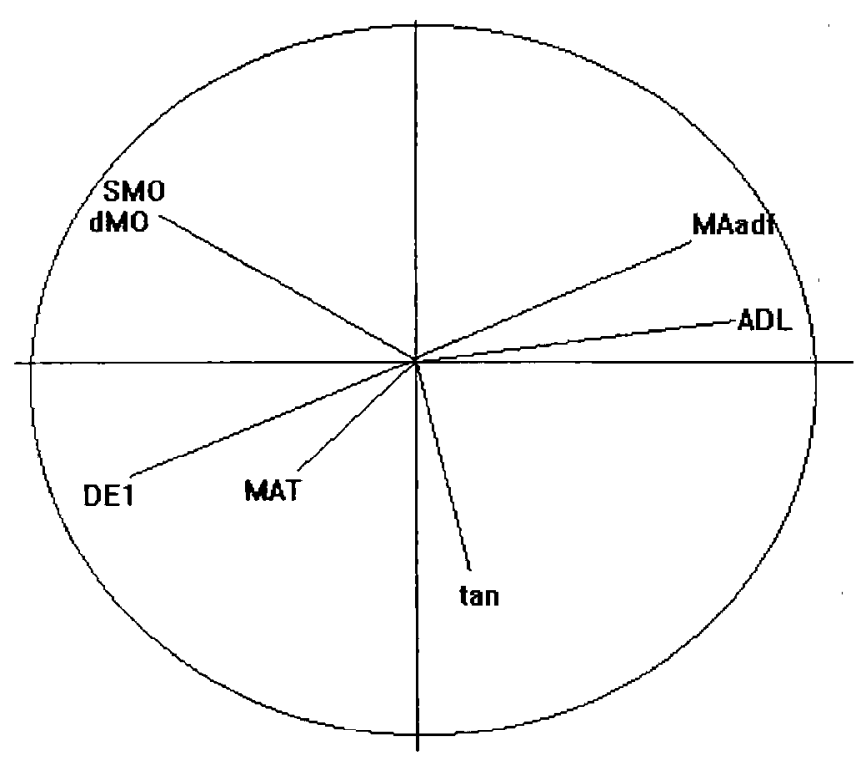

Figure 4 : cercle des corrélations. 


\section{La typologie}

La figure 5 présente la projection des 45 arbustes fourragers de cette étude sur le plan principal de l'ACP.

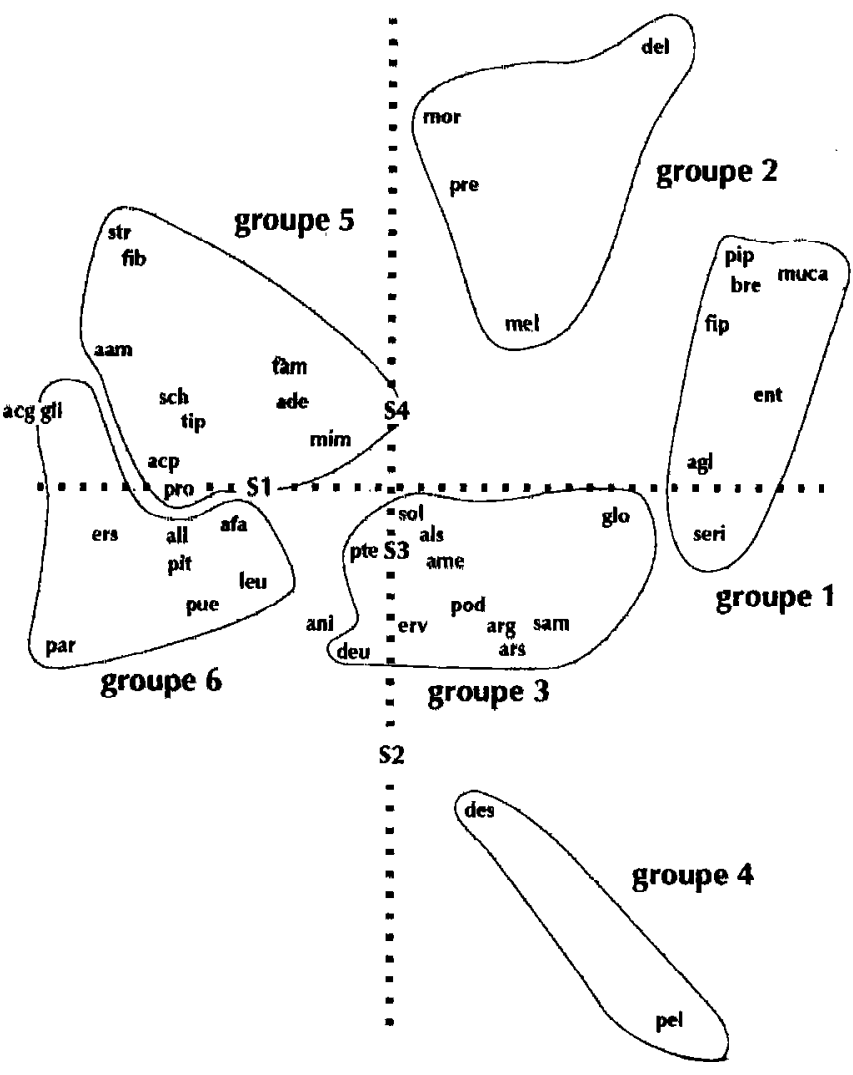

Figure 5 : projection des observations sur le plan principal.

L'échantillon de Sesbania grandiflora, de par la grande dégradabilité enzymatique in vitro de sa matière organique et de par sa forte teneur en azote très soluble chimiquement ou par voie enzymatique, est apparu très isolé sur le plan principal. En conséquence, afin de ne pas déséquilibrer la projection des observations, cette espèce a été exclue de l'analyse.

Six groupes ont été identifiés à partir du dendogramme de l'analyse. Leurs critères et les espèces concernées sont les suivants :

- groupe 1 :

Espèces (7) : Entada phaseolö̈des (ent), Mucuna platyphylla (muc), Serianthes sachetae (ser), Aglaia elaeagnoidea (agl), Ficus prolixa (fip), Pipturus incanus (pip), Breynia disticha (bre).

Caractérisation du groupe 1 :

\begin{tabular}{lcccc}
\hline & SMO & dMOgt & DE1 & MAadf \\
& $\%$ MS & $\%$ MS & \% MAT & \% MS \\
\hline caractérisation & faible & faible & faible & élevée \\
moyenne & 34,3 & 41,2 & 12,9 & 9,4 \\
écart-type & 9,3 & 4,7 & 5,1 & 2,5 \\
\hline
\end{tabular}

Le groupe 1 se caractérise aussi par sa richesse en fibres (ADL > 30 p. $100 \mathrm{MS}$ ).

\section{- groupe 2 :}

Espèces (4) : Melochia odorata (mel), Premna integrifolia (pre), Morinda citrifolia (mor), Delarbrea paradoxa (del).

Caractérisation du groupe 2 :

\begin{tabular}{lcccc} 
& SMO & dMOgt & DE1 & MAadf \\
& $\% \mathrm{MS}$ & $\% \mathrm{MS}$ & $\% \mathrm{MAT}$ & \% MS \\
\cline { 2 - 5 } caractérisation & élevée & élevée & faible & élevée \\
moyenne & 56,4 & 57,7 & 13,3 & 8,5 \\
écart-type & 6,1 & 5,7 & 3,6 & 1,1 \\
\hline
\end{tabular}

- groupe 3 :

Espèces (11): Samanea saman (sam), Archidendropsis granulosa (arg) et streptocarpa (ars), Pterocarpus indicus (pte), Erythrina variegata (erv), Desmodium umbellatum (des), Albizia serianthes (als), Solanum mauritianum (sol), Acacia mearsii (mea), Podonephelium homei (pod), Glochidion billardieri (glo).

Caractérisation du groupe 3 :

\begin{tabular}{lcccc}
\hline & SMO & dMOgt & DE1 & MAadf \\
& $\%$ MS & $\%$ MS & $\%$ M $\Lambda \mathrm{T}$ & $\%$ MS \\
\hline caractérisation & faible & faible & variable & variable \\
moyenne & 41,9 & 40,8 & 26,4 & 4,0 \\
écart-type & 5,0 & 2,7 & 7,5 & 1,6 \\
\hline
\end{tabular}

- groupe 4 :

Espèces (2) : Desmanthus virgatus (des), Peltoforum ferrugineum (pel).

Ce groupe se caractérise essentiellement par sa richesse en tanins précipitants $(>)$ et dans une moindre mesure par sa pauvreté en MAT.

- groupe 5 :

Espèces (11) : Schlenitzia insularum (sch), Adenanthera pavonina (ade), Ficus sp. (fis), Tipuana tipu (tit), Prosopis pallida (pro), Strobilopanax macrocarpae (str), Acacia ampliceps (amp), Acacin nilotica (ani), Tamarindus indica (tam), Mimosa invisa (mim), Acalypha pancheriana (acp)

Caractérisation du groupe 5 :

\begin{tabular}{lcccc}
\hline & SMO & dMOgt & DE1 & MAadf \\
& $\%$ MS & $\%$ MS & $\%$ MAT & $\%$ MS \\
\hline caractérisation & élevée & élevée & moyenne & variable \\
moyenne & 60,4 & 55,8 & 25,0 & 1,9 \\
écart-type & 8,9 & 3,7 & 6,7 & 1,0
\end{tabular}

Le groupe 5 se caractérise de plus par des teneurs en ADL inférieures à 19 p. $100 \mathrm{MS}$ ).

- groupe 6 :

Espèces (9) : Acalypha grandis (acg), Pueraria lobata (pue), Albizia lebbeck (all), Erythrina sp. (ers), Gliricidia sepium (gli), Acacia farnesiana (far), Leucaena leucocephala (leu), Pithecellobium dulce (pit), Parkinsonia aculeata (par). 
Caractérisation du groupe 6 :

\begin{tabular}{lcccc}
\hline & SMO & dMOgt & DE1 & MAadf \\
& \% MS & \% MS & \% MAT & $\%$ MS \\
\hline caractérisation & élevée & élevée & variable & variable \\
moyenne & 57,9 & 55,9 & 32,7 & 2,4 \\
écart-type & 7,7 & 4,9 & 11,1 & 0,5 \\
\hline
\end{tabular}

Il faut également noter que les teneurs en MAT de ces espèces sont relativement élevées (MAT > 20 p. $100 \mathrm{MS}$ ). Les teneurs en ADL sont inférieures à 15 p. 100 MS.

\section{Les ubservations supplémentaires}

Les Fabacées (S1) et les Euphorbiacées (S4) sont à rapprocher du groupe 5. Par contre les Mimosacées (S3) et les Caesalpiniacées (S2) sont mal représentées sur le plan principal, ce qui reflète l'hétérogénéité de composition au sein d'une même famille.

\section{DISCUSSION}

Cette étude a pour objet de mettre en évidence les espèces ligneuses potentiellement intéressantes sur le plan nutritionnel. Elle est basée uniquement sur leur composition chimique et leurs dégradabilités enzymatiques ou in vitro. Les factcurs pédoclimatiques (type de sol, pluviométrie, résistance à la sécheresse), agronomiques (possibilité de bouturage, présence d'épines, vitesse de croissance, production au cours de l'année, restitution d'azote dans le sol...) et d'utilisation par l'animal (appétabilité, ingestibilité...) ne sont pas abordés.

\section{Critères de choix}

Il n'y a pas actuellement de méthode fiable de prédiction de la valeur énergétique (UF) et de la valeur azotée (PDI) des ligneux fourragers. Leur valeur alimentaire ne sera donc approchée que par hiérarchie suivant leur composition chimique et leurs dégradabilités enzymatiques et in vitro.

Hormis leur intérêt agronomique, les arbustes fourragers pcuvent représenter une ressource protéique conséquente, notamment en saison sèche. C'est donc d'abord sur leur valeur azotée que les espèces seront sélectionnées. Cependant, du fait probablement de la grande diversité botanique des ligneux fourragers, la fermentescibilité de l'azote est très variable (distribution différente des matières azotées partiellement bloquées au niveau des parois indigestibles) $(2,14,22)$. La simple teneur en MAT est donc insuffisante pour apprécier la valeur azotée des ligneux fourragers. C'est pourquoi on s'attachera d'avantage à la dégradabilité de l'azote (DE1) et à sa disponibilité (MAnadf) qui donnent une idée beaucoup plus fiable de l'utilisation réelle par l'animal.

Le second critère est la valeur énergétique. Tout comme pour la valeur protéique, la digestibilité in vitro de la matière organique (dMOgt) et la solubilité enzymatique (SMO), inversement proportionnelles à la teneur en fibres, seront des critères privilégiés par rapport aux teneurs en éléments bruts. Les teneurs en fibres (en particulier NDF et $\mathrm{ADL}$ ), corrélées négativement avec la valeur énergétique d'un fourrage (22), interviendront comme éléments explicatifs. Il faut d'ailleurs noter que, si la teneur en NDF des ligneux fourragers est plus faible que chez les espèces herbacées, il n'en est pas de même pour leur teneur en lignines (14). Ceci explique, au moins en partie, la moindre solubilité de l'azote des fourrages ligneux comparativement à celle des herbacées (4).

Les facteurs antinutritionnels (tanins) constituent le troisième critère. Comme pour les lignines, leur teneur et leur répartition selon l'origine botanique de l'arbuste (famille, espèce) sont très variables (23), d'où leur intérêt pour discriminer les ligneux fourragers. Toutefois, il faut noter que la présence de tanins dans les feuilles des arbustes fourragers peut être bénéfique pour leur valeur alimentaire, qu'ils soient ingérés seuls ou en complément d'autres fourrages. Ils peuvent en effet favoriser la formation de protéines bypass et, ainsi, permettre le passage direct dans l'intestin de protéines digestibles (22). Des études complémentaires sur la nature et le mode d'action des tanins sont néanmoins nécessaires pour mieux interpréter la valeur azotée des arbustes fourragers.

Les teneurs en matières minérales (MM, $\mathrm{Ca}$ et $\mathrm{P})$ et en matières grasses (MG), qui jouent un faible rôle sur la valeur énergétique des fourrages, seront prises en compte en dernier.

-. En résumé, dans des conditions de pâturage de parcours extensifs (ration de base riche en fourrages lignocellulosiques), les meilleures espèces ligneuses sont celles riches en matières azotées fermentescibles (DE1 élevée, MAadf faible) et pauvres en fibres indigestibles (ADL faible, dMOgt et SMO élevées).

\section{Espèces ligneuses "à retenir"}

Les espèces du groupe 6 entrent dans le cadre défini ci-dessus : elles sont riches en matières azotées disponibles (MAT > 20 p. 100 MS, MAadf < 15 p. 100 MAT) avec des dégradabilités élevées $(\mathrm{SMO}>50$ p. 100; DE1 > 25 p. $100 \mathrm{MAT}$ ), pauvres en fibres et pauvres en tanins. Il n'est pas surprenant d'y trouver trois espèces largement exploitées en zones tropicales : Gliricidia sepium, Leucaena leucocephala et Albizia lebbeck $(11,17,25)$. En Nouvelle Calédonie, Acalypha grandis 'avait d'autre part déjà été signalée comme un arbuste fourrager à multiplier sur les îles Loyauté (10). Par leur teneur en éléments bruts et leur SMO (78 p. $100 \mathrm{MS}$ !), il faut également souligner la qualité des feuilles de Sesbania grandiflora, bien qu'elles ne semblent pas être consommées par les animaux (15).

Pour les mêmes raisons, les espèces du groupe 5 sont intéressantes sur le plan nutritionnel bien qu'elles soient moins riches en protéines disponibles. Elles sont notamment pauvres en NDF (NDF < 50 p. $100 \mathrm{MS}$ ) et, par conséquent, leur SMO est élevée (SMO > 55 p. $100 \mathrm{MS}$ ). Enfin, si Acacia ampliceps' a une teneur très faible en MAT (10,8 p. $100 \mathrm{MS}$ ), en revanche la dégradabilité enzymatique de l'azote est élevée (DE1 = 35,7 p. $100 \mathrm{MAT})$.

Dans le groupe 3 , les espèces sont riches en protéines (MAT > 17,5 p. $100 \mathrm{MS}$ ). Cependant, leur intérêt est limité compte tenu de leur richesse en parois (NDF > 55 p. $100 \mathrm{MS}, \mathrm{ADL}>18$ p. 100 MS) et de leurs faibles dégradabilités enzymatiques (SMO < 40 p. 100 MS). Par sa tencur cn matic̀rcs azotées, Samanea saman paraît néanmoins prometteuse bien que sa valeur DE1 ne soit que de 19,4 p. 100 MAT.

\section{Espèces ligneuses "à écarter"}

Sur le plan nutritionnel, les espèces des groupes 1 et 2 ont peu d'intérêt parce qu'elles sont pauvres en matières azotées disponibles (MAT < 15 p. $100 \mathrm{MS}$, teneur en MAadf élevée, DE1 $\leq$ 25 p. $100 \mathrm{MAT}$ ) et pauvres en énergie (NDF > 45 p. $100 \mathrm{MS}$, $\mathrm{dMOgt}<40$ p. 100) (groupe 1). Parmi les espèces des groupes 1 et 2 , les moins intéressantes sur le plan de la valeur nutritive sont Ficus prolixa et Pipturus incanus.

Les deux espèces du groupe 4 sont particulièrement riches en tanins précipitants. On peut citer en exemple la variété locale de Desmanthus virgatus (tanpt $=7,35$ p. $100 \mathrm{MS}$ ). Ces espèces sont peu intéressantes sur le plan nutritionnel. 


\section{Limites et prolongements de l'étude}

Les teneurs en éléments bruts résultent de l'analyse d'un seul échantillon par espèce. Leur valeur statistique est donc limitée. De la même façon, les récoltes ont été faites sur une courte période de l'année (deux mois). Non seulement les stades phénologiques des ligneux arbustifs étaient différents (tableau I), mais leur teneur en éléments bruts aurait sûrement varié pour une autre saison, dans des proportions qu'il conviendrait de déterminer. Des études menées en Afrique et en Australie $(16,18,25)$ montrent d'ailleurs des variations de composition au cours de l'année ou selon les types de sols.

Aussi, hien que l'enquête de terrain et l'analyse des échantillons prélevés illustrent la démarche relativement rapide qui peut être adaptée pour une première identification des espèces fourragères ayant la meilleure valeur nutritionnelle dans une région donnée, convient-il de préciser et de consolider les résultats notamment en matière :

- de production et de disponibilité saisonnière des fourrages - d'appétabilité

- d'utilisation digestive.

\section{CONCLUSION}

Cette étude, réalisée sur 45 espèces arbustives, a permis de mettre en évidence six groupes de ligneux fourragers caractérisés par leurs teneurs en éléments bruts et leurs dégradabilités enzymatiques. Les espèces présentant des teneurs élevées en matières azotées dégradables et faibles en fibres et tanins sont les plus intéressantes sur lc plan nutritionncl. Il s'agit notamment de Gliricidia sepium, de Leucaena leucocephala, d'Acalypha grandis, de Samanea saman, d'Albizia lebbeck, d'Erythrina sp., d'Acacia farnesiana et de Sesbania grandiflora. Les données pour Desmanthus virgatus, Parkinsonia aculeata et, dans une moindre mesure, Acacia ampliceps sont en revanche décevantes.

Mais cette étude n'est qu'une étape dans notre programme de recherches, l'objectif final étant l'implantation chez les éleveurs d'espèces adaptées à leurs conditions pédoclimatiques, valorisées par les ruminants et permettant le maintien de la fertilité des sols. Aussi des travaux complémentaires sont-ils actuellement menés en Nouvelle Calédonie pour déterminer la productivité, le pouvoir de fixation de l'azote et l'appétabilité de ligneux arbustifs. Des essais de gestion de cette ressource fourragère sont également à l'étude.

D'autre part, des études complémentaires sur la nature et le mode d'action des lignines et des tanins semblent nécessaires compte tenu de leur diversité et de leur importance dans la valeur nutritive des arbustes fourragers. Ce travail doit notamment s'intéresser à leur identification (méthodes chimiques ou enzymatiques) et à leur caractérisation. En outre, cette étape permettra d'établir plus précisément des équations de prédiction de la valeur alimentaire des ligneux fourragers.

\section{Remerciements}

Ce travail a été réalisé dans le cadre du programme de la CEEDGXII/ST2-0215. Aussi tenons-nous à adresser nos sincc̀rcs rcmerciements aux trois laboratoires (laboratoire d'analyse des aliments du bétail, Nouméa; laboratoire d'alimentation CIRADEMVT de Maisons-Alfort ; Institut für Tierernährung, Universität Hohenheim) qui nous ont aidés dans cette étude.
BIBLIOGRAPHIE

1. AFNOR, 1980. Recueil de normes françaises des méthodes générales d'analyse des produits agro-alimentaires. Chimie-microbiologie-analyse sensorielle. Paris, France AFNOR, 278 p.

2. ARBELOT B., 1993. Prédiction de la valeur nutritive des feuilles de fourrages ligneux tropicaux. Mémoire DEA, université d'Aix Marseille II et III/Paris-Grignon INA, France, $25 \mathrm{p}$.

3. AUFRERE J., DEMARQUILLY C., 1989. Predicting organic matter digestibility of forage by two pepsin-cellulase methods. In: Proceedings of the 16th International Grassland Congress, Nice, France, 4-11 octobre 1989, p. 877-878.

4. AUFRERE J., GUERIN H., 1996. Critical review of chemical and enzymatic methods for the estimation of nutritive value in roughages. Ann 7ont., 45, suppl. : 11-28

5. AUFRERE J., MICHALET-DOREAU B., 1990. Nouvelles méthodes d'estimation de la valeur alimentaires des fourrages. II. Méthodes enzymatiques. Fourrages, 122 : 203-217.

6. BIPEA, 1976-81. Recueil des méthodes d'analyse des Communautés européennes. Paris, France, ITCF.

7. BRAY R.A., 1994. The Leucaena psyllid. In : Forage tree legumes in tropical agriculture. Wallingford, United Kingdom, CAB International, $389 \mathrm{p}$.

8. CHAZEAU J., CAPART I., BONNET de LARBOGNE L., 1992. Amélioration de l'état phytosanitaire du faux-mimosa Leucaena leucocephala en Nouvelle Calédonie par le renforcement du complexe des ennemis naturels du psylle ravageur Heteropsylla cubana. Nouméa, Nouvelle Calédonie, ORSTOM, 24 p. (Sciences de la Vie, Zoologie appliquée, Conventions ${ }^{\circ}$ 6)

9. CIRAD EMVT, 1994. Caractéristiques et valeurs alimentaires des fourrages en Nouvelle Calédonie. Nouméa, Nouvelle Calédonie, CIRADEMVT, $150 \mathrm{p}$

10. CORNIAUX C., 1991. Recherche sur Maré des espèces ayant un intérêt fourrager. Nouméa, Nouvelle Calédonie, CIRAD-Elevage, $11 \mathrm{p}$.

11. DEVENDRA C., 1992. Nulrilional polential of fodder trees and shrubs as protein sources in ruminant nutrition. In : Speedy and Pugliese eds, Legume trees and other fodder trees as protein sources for livestock. Rome, Italy, FAO, p. 95-113.

12. DURAND N., 1993. Identification d'espèces ligneuses arbustives utilisables par les ruminants dans les parcours extensifs en Nouvelle Calédonie. Nouméa, Nouvelle Calédonie, CIRAD-EMVT, 8 p. +45 fiches.

13. GRILLET C., VILLENEUVE F., 1994. Les tanins dans les fourrages ligneux. Ch. VI, 34 p. In : Guerin H. éd., Valeur alimentaire des fourrages ligneux consommés par les ruminants en Afrique centrale et de l'Ouest. Maisons-Alfort, France, CIRAD-EMVT, 393 p.

14. GUERIN H. éd., 1994. Valeur alimentaire des fourrages ligneux consommés par les ruminants en Afrique centrale et de l'Ouest. Commission des Communautés européennes, DGXII, programme ST2.A/89/215.F, Maisons-Alfort, France, CIRAD-EMVT, 393 p.

15. GUTTERIDGE R.C. 1994. Other species of multipurpose forage tree legumes. In : Forage tree legumes in tropical agriculture. Wallingford, United Kingdom, CAB International, $389 \mathrm{p}$.

16. GUTTERIDGE R.C., SHELTON H.M., 1994. The role of forage tree legumes in cropping and grazing systems. In : Forage tree legumes in tropical agriculture. Wallingford, United Kingdom, CAB International, $389 \mathrm{p}$.

17. HUMPHREYS L.R., 1980. A guide to better pastures for the tropics and sub-tropics, 4th ed. Australia, Wright Stephenson \& Co, 95 p.

18. ICKOWICZ A., 1995. Approche dynamique du bilan fourrager appliqué à des formations pastorales du Sahel tchadien. Thèse doc., université Paris XII, Créteil, France, $471 \mathrm{p}$.

19. INRA, 1989. Ruminant Nutrition. Jarrige R. ed. London, United Kingdom, John Libbey Eurotext, 389, p.

20. MASON V.L., 1969. Some observations on the distribution and origin of nitrogen in sheep faeces. J. agric. Sci. Camb., 73: 99-111.

21. MENKE H., STEINGASS H., 1988. Estimation of the energetic feed value obtained from chemical analysis and in vitro gas production using rumen fluid. Anim. Res. Devel., 28: 7-55. 
22. NORTON B.W., 1994. The nutritive value of tree legumes. In Forage tree legumes in tropical agriculture. Wallingford, United Kingdom, CAB International, $389 \mathrm{p}$.

23. NORTON B.W., 1994. Tree legumes as dietary supplements for ruminants. In : Forage tree legumes in tropical agriculture. Wallingford, United Kingdom, CAB International, $389 \mathrm{p}$.

24. PHILIPPEAU G., 1986. Comment interpréter les résultats d'une analyse en composantes principales. Paris, France, ITCF, $63 \mathrm{p}$.

\section{Summary}

Corniaux C., Durand N., Sarraihl J.M., Guerin H. Chemical components and enzyme and in vitro digestibility of woody shrubs used by ruminants in New Caledonian rangelands. I. Typology

This study, performed on 45 shrubs from New Caledonia, helped define six fodder shrub groups characterized by their chemical components and their enzyme and in vitro digestibility. Species with highly digestible crude protein contents and organic matter digestibility are the most interesting from a nutritional point of view. The best results are obtaincd with Gliricidia sepium, Leucaena leucocephala, Acalypha grandis, Samanea saman, Albizia lebbeck, Erythrina sp., Acacia farnesiana and Sesbania grandiflora. On the other hand, data on Desmanthus virgatus and, to a lesser degree, Acacia ampliceps are disappointing.

Key words: Browse plant - Protein value - Classification Chemical composition - Digestibility - Ruminant - Rangeland - New Caledonia.
25. SHELTON H.M., BREWBAKER J.L., 1994. Leucaena leucocephala the most widely used forage tree legume. In : Forage tree legumes in tropical agriculture. Wallingford, United Kingdom, CAB International, $389 \mathrm{p}$.

26. VAN SOEST P.J., WINE R.H., 1967. Use of detergents in the analysis of fibrous feeds. J. Ass. Off. Anal, 50: 50-55.

Reçu le 23.5.95, accepté le 24.6 .96

\section{Resumen}

Corniaux C., Durand N., Sarraihl J.M., Guerin H. Composición química y degradación enzimática e in vitro de las especies de arbustos leñosos utilizados por los rumiantes en los trayectos extensivos de Nueva Caledonia

Este estudio, realizado en 45 especies de arbustos recolectados en Nueva Caledonia, permitió demostrar seis grupos de forrages leñosos caracterizados por su contenido en elementos brutos y por su degradación enzimática e in vitro. En estas especies, el conlenido de materias nitrogenadas degradables y un alto grado de degradación de materia orgánica son los puntos de mayor interés nutricional. Se trata de Gliricidia sepium, de Leucaena leucocephala, de Acalypha grandis, de Samanea saman, de Albizia lebbeck, de Erythrina sp., de Acacia farnesiana y de Sesbania grandiflora. Por otro lado, los datos correspondientes a Desmanthus virgatus $y$, en menor grado, Acacia ampliceps no son satisfactorios.

Palabras clave : Planta de ramoneo - Valor proteinico Clasificación - Composición química - Digestibilidad Rumiante - Tierra de pastos - Nueva Caledonia. 